\title{
Retração em Argamassa de Revestimento: Uma Revisão da Literatura
}

\author{
Géssica Katalyne Bilcati'; Marienne do Rocio de Mello Maron da Costa²; Felipe Simionï; Pâmela \\ Albino $^{2}$; Roberto Luiz Dias ${ }^{2}$
}

\gessicak@utfpr.edu.br

1. Universidade Tecnológica Federal do Paraná, Guarapuava - PR, Brasil.

2. Universidade Federal do Paraná, Curitiba - PR, Brasil.

\section{Histórico do Artigo:}

Recebido em: 26 de março de $2020 \quad$ Aceito em: 13 de julho de $2020 \quad$ Publicado em: 31 de agosto de 2020

\begin{abstract}
Resumo: 0 grande número de publicações contendo dados experimentais sobre o fenômeno de retração que pode ocorrer em elementos de construção torna o trabalho dos pesquisadores cada vez mais complexo e extenso no que se refere à seleção de material bibliográfico para o embasamento da pesquisa. Dessa forma, foi realizada uma revisão bibliográfica com os temas retração de secagem e plástica em argamassas de revestimento, entre os anos de 2013 e 2018, utilizando os procedimentos do método MethodiOrdinatio para avaliar a atual conjuntura das pesquisas nesse tema. Apesar do crescente número de publicações, é notável a disparidade na quantidade de pesquisas entre os dois tipos de retração, sendo a grande maioria formada por retração por secagem. Além disso, estudos relacionados à retração plástica se mostraram menos relevantes no âmbito científico, aparecendo apenas em $12^{\circ}$ lugar no ranking de relevância, o que não condiz com a importância desse tipo de retração nas características do estado plástico e endurecido do material. Dessa forma, é de suma importância o preenchimento das lacunas de conhecimento existente e formulação de ensaios capazes de avaliar a retração do material no estado plástico.
\end{abstract}

Palavras-chave: Revisão bibliométrica, Retração de secagem, Retração plástica.

\section{Shrinkage in coating Mortar: A Literature Keview}

\begin{abstract}
The number of publications containing experimental data on shrinkage phenomenon of $\mathrm{n}$ building elements is high. In the search and selection of bibliographic material, this high number makes the work of researchers more complex and extensive. Thus, a bibliographic review, between the years 2013 and 2018, was carried out, with the topic drying shrinkage and plastic shrinkage in coating mortars. Using the procedures of the MethodiOrdinatio method, the current position of research on this topic was evaluated. Despite the growing number of researches, the disparity in the amount of research between the two types of shrinkage is notable. Most studies are about shrinkage by drying. In addition, studies related to plastic shrinkage were less relevant in the scientific field. These studies appear only in 12th place in the ranking of relevance, does not show the real importance of this type of shrinkage in the characteristics of the plastic and hardened state of the material. Thus, it is very important to fill the gaps in existing knowledge and develop tests capable of evaluating the shrinkage of the material in the plastic state.
\end{abstract}

Keywords: Bibliometric review, Drying shrinkage, Plastic shrinkage. 


\section{Retracción en Mortero de Recubrimiento: Una Revisión de la Literatura}

Resumen: El gran número de publicaciones que contienen datos experimentales sobre el fenómeno de retracción que pueden ocurrir en materiales de construcción hace que el trabajo de los investigadores sea cada vez más complejo y extenso con respecto a la selección de material bibliográfico para base teórica. Por lo tanto, se realizó una revisión bibliográfica con el tema del secado y la retracción plástica en morteros de revestimiento, entre los años 2013 y 2018, utilizando los procedimientos del método MethodiOrdinatio para evaluar la coyuntura actual de la investigación sobre este tema. A pesar del creciente número de publicaciones, la disparidad en la cantidad de investigación entre los dos tipos de retracción es notable, con la gran mayoría formada por retracción por secado. Además, los estudios relacionados con la retracción plástica fueron menos relevantes en el campo científico, apareciendo solo en el puesto 12 en la clasificación de relevancia, lo que no coincide con la importancia de este tipo de retracción en las características del plástico y el estado endurecido del material. Por lo tanto, es extremadamente importante llenar los vacíos en el conocimiento existente y formular pruebas capaces de evaluar la retracción del material en estado plástico.

Palabras clave: Revisión bibliométrica, Retracción por secado, Retracción plástica.

\section{INTRODUÇÃo}

Materiais cimentícios são largamente empregados no setor da construção civil devido à facilidade na execução, elevado desempenho e durabilidade, como também custo relativamente baixo, onde os produtos constituintes básicos apresentam grande disponibilidade. No entanto, argamassas e concretos à base de cimento Portland, são compostos frágeis e sensíveis a fissuras devido à baixa resistência à tração (MEHTA e MONTEIR0, 2008).

As fissuras dos compósitos cimentícios podem ocorrer antes da aplicação de carga, facilitando a penetração de agentes agressivos que podem causar degradação de concretos e argamassas, como resultado, a vida útil pode ser reduzida (LU, 2019).

As fissuras não estruturais das argamassas de revestimento são ocasionadas pelo fenômeno de retração devido às mudanças de umidade (que resultam em tensão superficial e capilar), transporte de água e reações químicas (KONSTANTIN e KOVLER, 2006).

A consistência adequada das argamassas à base de cimento Portland, requer frequentemente o uso de maior quantidade de água na mistura, que é necessário para o processo de hidratação. A perda da água incorporada, à medida que os materiais cimentícios endurecem, resultam em uma redução de volume conhecida como retração. A redução de volume que ocorre antes do compósito cimentício endurecer, é chamada de retração plástica, se a redução ocorre devido à perda de umidade após o endurecimento é conhecida por retração de secagem (SILVA, 2011).

Além da retração plástica e de secagem, materiais cimentícios podem apresentar retração autógena, térmica e química. A retração autógena ocorre devido ao consumo da água 
presente nos capilares em decorrência das reações de hidratação. Já a retração térmica se origina de parte do vapor formado pelo calor das reações exotérmicas de hidratação, assim quando a taxa de hidratação diminui, a temperatura diminui e ocorre a retração. Por fim, a retração química ocorre devido aos compostos hidratados terem menor volume que o material anidro (MELO NETO, 2002).

A principal preocupação em relação a retração em argamassas para revestimento é a formação de fissuras, que podem ocorrer tanto no estado plástico como no endurecido (SEGAT, 2005). Para o entendimento do mecanismo dos tipos de retração nas propriedades das argamassas, é necessário monitorar a variação dimensional em todas as fases do processo de produção.

No Brasil a norma regulamentadora NBR 15261 (ABNT, 2005), que versa sobre a retração de argamassas, recomenda a determinação de variação dimensional (retração ou expansão linear) a partir da moldagem de corpos de prova prismáticos. As leituras de variação dimensional são realizadas com o auxílio de um aparelho comparador, sendo recomendada a leitura nas idades 1,7 e 28 dias.

Entretanto, estudos realizados por Bastos et al. (2005) evidenciaram que uma parcela significativa da retração ocorre no estado plástico, o que é negligenciado pela norma. Outro resultado obtido no estudo mostrou que as argamassas com os maiores valores de retração no estado endurecido não correspondem, necessariamente, às argamassas com os maiores resultados de retração plástica. Dessa forma, ao avaliar a retração das argamassas somente no estado endurecido, pode interferir no desempenho e até mesmo reduzir a vida útil do material (BASTOS et al., 2005).

Considerando a relevância do estudo do fenômeno de retração em argamassas de revestimento, o presente artigo tem a finalidade de fazer levantamento qualitativo-quantitativo de pesquisas anteriores, realizadas entre os anos de 2013 e 2018, que abordam a retração no estado plástico e endurecido, assim como a avaliação de normas e metodologias de ensaios empregadas para a medição do fenômeno de retração, a fim de fornecer um panorama geral e comparativo da retração nos dois estágios.

\section{MATERIAL E MÉTODOS}

A metodologia MethodiOrdinatio proposta por Pagani et al. (2015) é uma ferramenta multicriterial de decisão, baseada no fator de impacto, número de citações e ano de publicação. 
0 raciocínio sobre essas três variáveis gera o InOrdinatio, cujo índice indica a relevância científica do artigo. A partir deste índice, é possível classificar os trabalhos individualmente. Os procedimentos desta pesquisa exploratória no qual é gerado um portfólio são descritas a seguir:

\section{Estabelecendo a intenção da pesquisa proposta}

Os eixos estabelecidos para construção do portfólio bibliográfico foram: retração (Shrinkage), argamassas de revestimento (coattingmortar e renderingmortar) e retração plástica (Plasticshrinkage), respectivamente. A pesquisa foi delimitada pelo ano de publicação dos artigos, compreendidos entre 2013 e 2018. Foi utilizado o software Mendeley como gerenciador de referências para a coleta e armazenamento dos dados.

As bases de dados selecionadas foram Science Direct e Web of Science. A escolha das bases fundamentou-se no grande volume de publicações obtido com as palavras-chave escolhidas. Além disso, foi verificado que a quantidade de publicações em outras bases era inferior às escolhidas, e muitos dos títulos contidos já haviam sido localizados nas bases de dados citadas. Por fim, além das dificuldades apontadas, essas bases não apresentaram consistência durante as buscas, trazendo resultados bastante divergentes a cada nova busca.

Após definidas as bases de dados, realizou-se a busca definitiva, resultando em um total de 1932 artigos, conforme mostrado na Tabela 1.

Tabela 1. Artigos encontrados na pesquisa nas bases de dados

\begin{tabular}{|c|c|c|c|}
\hline \multirow{3}{*}{ Palavras-chave } & \multicolumn{2}{|l|}{ BASE DE DAD0S } & \multirow{3}{*}{ TOTAI } \\
\hline & SCIENCE DIRECT & WEB OF SCIENCE & \\
\hline & \multicolumn{2}{|c|}{ TOTAL DE ARTIGOS ENCONTRADOS } & \\
\hline Shrinkage AND “Coatingmortar” & 1598 & 13 & 1611 \\
\hline Shrinkage AND "Renderingmortar" & 303 & 12 & 315 \\
\hline $\begin{array}{l}\text { "Plastic shrinkage" AND "Coating } \\
\text { mortar" }\end{array}$ & 01 & 0 & 01 \\
\hline $\begin{array}{l}\text { "Plastic shrinkage" AND "Rendering } \\
\text { mortar" }\end{array}$ & 05 & 0 & 05 \\
\hline
\end{tabular}

Fonte: Autores (2020).

\section{Procedimento de filtragem}

0 procedimento de filtragem foi realizado de maneira a compatibilizar os resultados obtidos em ambas as bases de dados, sendo analisados individualmente aplicando os seguintes filtros: eliminando a duplicidade (facilmente realizada usando o gerenciador de referência); 
artigos cujo título não estava relacionado com a área; capítulos de libros ou conferências (não têm fator de impacto). Por fim, as palavras-chave e os resumos foram analisados para verificar se 0 artigo estava, ou não, relacionado com a área. Na tabela 2 é mostrado o volume de artigos eliminados em cada procedimento de filtragem.

Tabela 2. Procedimento de filtragem dos artigos estudados.

\begin{tabular}{llll}
\hline Procedimentos de filtragem usados & Artigos selecionados & $\begin{array}{c}\text { Artigos } \\
\text { excluídos }\end{array}$ & (\%) \\
\hline Total bruto de artigos & 1932 & & 100 \\
Artigos de capítulos de livros/ conferências & 546 & 28,26 \\
$\begin{array}{l}\text { Duplicidade de artigos } \\
\text { Número de artigos excluídos após leitura do }\end{array}$ & 522 & 27,02 \\
título & 777 & 40,22 \\
Número de artigos excluídos após leitura do & 61 & 3,16 \\
resumo & & 98,46 \\
Total de artigos descartados & 1906 & 1,44 \\
Total de artigos selecionados para compor 0 & 26 & & \\
portfólio & & & \\
\hline
\end{tabular}

Fonte: Autores (2020).

\section{Identificação do fator de impacto, ano de publicação e número de citações}

Através do procedimento de filtragem realizado, restaram 26 artigos para compor o escopo da pesquisa. 0 passo subsequente foi classificar os artigos usando a Equação In0rdinatio (1).

InOrdinatio $=\left(\frac{I F}{1000}\right)+(\alpha *(10-($ ResearchYear - PublishYear $)))+(C i)$

A pesquisa foi realizada tendo como base o fator de impacto e número de citação, utilizando o gerenciador de referência JabRef, o qual gerou automaticamente uma lista de artigos com o nome dos autores, título do trabalho, nome da revista, ano de publicação, tipo de publicação e URL. 0 número de citações foi extraído manualmente de Google Acadêmico. 0 fator de impacto também foi obtido manualmente através da lista fornecida pelo Scopus2.

Por fim, foi obtido o ranking dos artigos usando o InOrdinatio, com a identificação de parâmetros, no qual 26 artigos foram classificados do maior para o menor Índice Ordinatio 
(In0rdinatio) usando valores alfa $(\alpha)$ de 10.0 valor de alfa $(\alpha)$ igual 10 representa que 0 ano de publicação do artigo é um fator relevante para a pesquisa (PAGANI et al., 2015).

\section{RESULTADOS E DISCUSSÃ0}

A seguir serão apresentados os resultados, avaliação e discussão sobre a leitura sistemática e análise dos artigos localizados nas bases de dados.

\section{Análise descritiva e metodológica dos artigos selecionados}

Através do método Methodi0rdinatio foi possível classificar os artigos mais relevantes sobre retração em argamassas de revestimento. 0 ranking dos artigos selecionados para discussão é mostrada na tabela 3.

Tabela 3. Ranking dos artigos pelo Methodi Ordinatio

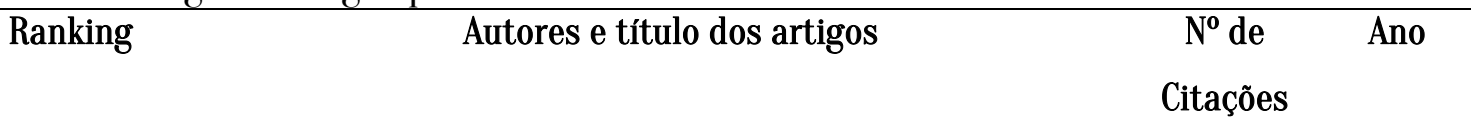

\begin{tabular}{|c|c|c|c|}
\hline $1^{0}$ & $\begin{array}{l}\text { Tan, K.H. and Du, H. Use of waste glass as sand in mortar: } \\
\text { Part I - Fresh, mechanical and durability properties. }\end{array}$ & 94 & 2013 \\
\hline $2^{0}$ & $\begin{array}{l}\text { Mobili, A., Belli, A., Giosuè, C., Bellezze, T. and Tittarelli, F. } \\
\text { Metakaolin and fly ash alkali-activated mortars } \\
\text { compared with cementitious mortars at the same } \\
\text { strength class. }\end{array}$ & 36 & 2016 \\
\hline $3^{\circ}$ & $\begin{array}{l}\text { Joshaghani, A., Balapour, M. and Ramezanianpour, A.A. } \\
\text { Effect of controlled environmental conditions on } \\
\text { mechanical, microstructural and durability properties of } \\
\text { cement mortar. }\end{array}$ & 16 & 2018 \\
\hline $4^{0}$ & $\begin{array}{l}\text { Kabeer, K.S.A. and Vyas, A.K. Utilization of marble powder } \\
\text { as fine aggregate in mortar mixes. }\end{array}$ & 8 & 2018 \\
\hline $5^{0}$ & $\begin{array}{l}\text { Bao, Y., Meng, W., Chen, Y., Chen, G. and Khayat, K.H. } \\
\text { Measuring mortar shrinkage and cracking by pulse pre- } \\
\text { pump Brillouin optical time domain analysis with a } \\
\text { single optical fiber. }\end{array}$ & 38 & 2015 \\
\hline $6^{\circ}$ & $\begin{array}{l}\text { Ralegaonkar, R., Gavali, H., Aswath, P. and Abolmaali, S. } \\
\text { Application of chopped basalt fibers in reinforced } \\
\text { mortar: A review }\end{array}$ & 4 & 2018 \\
\hline
\end{tabular}




\begin{tabular}{|c|c|c|c|}
\hline $7^{0}$ & $\begin{array}{l}\text { Ergenç, D., Gómez-Villalba, L.S. and Fort, R. Crystal } \\
\text { development during carbonation of lime-based mortars } \\
\text { in different environmental conditions. }\end{array}$ & 3 & 2018 \\
\hline $8^{0}$ & $\begin{array}{l}\text { Bayat, A., Hassani, A. and Yousefi, A. Effects of red mud } \\
\text { on the properties of fresh and hardened alkali-activated } \\
\text { slag paste and mortar. }\end{array}$ & 1 & 2018 \\
\hline $9^{\circ}$ & $\begin{array}{l}\text { Li, L., Wang, R. and Lu, Q. Influence of polymer latex on } \\
\text { the setting time, mechanical properties and durability of } \\
\text { calcium sulfoaluminate cement mortar. }\end{array}$ & 1 & 2018 \\
\hline $10^{\circ}$ & $\begin{array}{l}\text { Abraham, S.M. and Ransinchung, G. Influence of RAP } \\
\text { aggregates on strength, durability and porosity of } \\
\text { cement mortar. }\end{array}$ & 0 & 2018 \\
\hline $11^{0}$ & $\begin{array}{l}\text { Beushausen, H. and Arito, P. The influence of mix } \\
\text { composition, w/b ratio and curing on restrained } \\
\text { shrinkage cracking of cementitious mortars. }\end{array}$ & 0 & 2018 \\
\hline $12^{\circ}$ & $\begin{array}{l}\text { Fuller, A., Stegmaier, M., Schulz, N., Menke, M., } \\
\text { Schellhorn, H., Knödler, F., Maier, J. and Scheffknecht, G. } \\
\text { Use of wood dust fly ash from an industrial pulverized } \\
\text { fuel facility for rendering. }\end{array}$ & 0 & 2018 \\
\hline $13^{0}$ & $\begin{array}{l}\text { Le, H.V., Moon, D. and Kim, D.J. Effects of ageing and } \\
\text { storage conditions on the interfacial bond strength of } \\
\text { steel fibers in mortars. }\end{array}$ & 0 & 2018 \\
\hline $14^{0}$ & $\begin{array}{l}\text { Penacho, P., de Brito, J. and Rosário Veiga, M. Physico- } \\
\text { mechanical and performance characterization of } \\
\text { mortars incorporating fine glass waste aggregate. }\end{array}$ & 39 & 2014 \\
\hline $15^{\circ}$ & $\begin{array}{l}\text { Chen, Z. and Poon, C.S. Comparative studies on the effects } \\
\text { of sewage sludge ash and fly ash on cement hydration } \\
\text { and properties of cement mortars. }\end{array}$ & 8 & 2017 \\
\hline $16^{0}$ & $\begin{array}{l}\text { Ling, T.-C. and Poon, C.S. Spent fluorescent lamp glass as } \\
\text { a substitute for fine aggregate in cement mortar. }\end{array}$ & 5 & 2017 \\
\hline $17^{\circ}$ & $\begin{array}{l}\text { Pan, Z., Tao, Z., Murphy, T. and Wuhrer, R. High } \\
\text { temperature performance of mortars containing fine } \\
\text { glass powders. }\end{array}$ & 5 & 2017 \\
\hline $18^{0}$ & $\begin{array}{l}\text { Lucas, J., de Brito, J., Veiga, R. and Farinha, C. The effect } \\
\text { of using sanitary ware as aggregates on rendering } \\
\text { mortars' performance. }\end{array}$ & 13 & 2016 \\
\hline $19^{\circ}$ & $\begin{array}{l}\text { Argane, R., Benzaazoua, M., Hakkou, R. and Bouamrane, A. } \\
\text { A comparative study on the practical use of low sulfide }\end{array}$ & 12 & 2016 \\
\hline
\end{tabular}




\begin{tabular}{|c|c|c|c|}
\hline & $\begin{array}{l}\text { base-metal tailings as aggregates for rendering and } \\
\text { masonry mortars. }\end{array}$ & & \\
\hline $20^{\circ}$ & $\begin{array}{l}\text { Oliveira, R., de Brito, J. and Veiga, R. Reduction of the } \\
\text { cement content in rendering mortars with fine glass } \\
\text { aggregates. }\end{array}$ & 19 & 2015 \\
\hline $21^{\circ}$ & $\begin{array}{l}\text { Aydın, S. A ternary optimisation of mineral additives of } \\
\text { alkali activated cement mortars. }\end{array}$ & 39 & 2013 \\
\hline $22^{\circ}$ & $\begin{array}{l}\text { Kim, H., Ha, K. and Lee, H. Internal-curing efficiency of } \\
\text { cold-bonded coal bottom ash aggregate for high- } \\
\text { strength mortar. }\end{array}$ & 6 & 2016 \\
\hline $23^{\circ}$ & $\begin{array}{l}\text { Duran, A., Navarro-Blasco, I., Fernández, J. and Alvarez, J. } \\
\text { Long-term mechanical resistance and durability of air } \\
\text { lime mortars with large additions of nanosilica. }\end{array}$ & 20 & 2014 \\
\hline $24^{\circ}$ & $\begin{array}{l}\text { da Silva, A.M., de Brito, J. and Veiga, R. Incorporation of } \\
\text { fine plastic aggregates in rendering mortars. }\end{array}$ & 18 & 2014 \\
\hline $25^{\circ}$ & $\begin{array}{l}\text { Anna, A. and Giuseppe, C. The water transfer properties } \\
\text { and drying shrinkage of aerial lime-based mortars: an } \\
\text { assessment of their quality as repair rendering } \\
\text { materials. }\end{array}$ & 10 & 2014 \\
\hline $26^{\circ}$ & $\begin{array}{l}\text { Muhammad, B. and Ismail, M. Performance of } \\
\text { hydrocarbon particles on the drying shrinkage of cement } \\
\text { mortar. }\end{array}$ & 3 & 2013 \\
\hline
\end{tabular}

Fonte: Autores (2020).

$\mathrm{Na}$ análise por periódico, a revista Construction and Building Materials se destaca com 15 publicações sobre o tema, seguido da revista Journal of Cleaner Production com 4 e Cement and Concrete Composites, com 2 publicações. A tabela 4 evidencia o número de artigos por revista para os todos os artigos estudados:

Tabela 4. Número de artigos por periódico

\begin{tabular}{lcc}
\hline \multicolumn{1}{c}{ Revistas } & Frequência & \% \\
\hline Construction and Building Materials & 15 & 57,7 \\
Journal of Cleaner Production & 4 & 15,4 \\
Cement and Concrete Composites & 2 & 7,7 \\
Cement and Concrete Research & 1 & 3,84 \\
Materials Letters & 1 & 3,84
\end{tabular}


Materials Characterization

Materials \& Design

Environmental Earth Sciences
3,84

3,84

3,84

Fonte: Autores (2020).

\section{Análise dos fatores que influenciam na retração em argamassas}

No estudo sobre retração por secagem realizada por Tan e Du (2013), os autores destacaram que a redução dos valores médios de retração por secagem é influenciada pela quantidade de água empregada na mistura, capacidade de absorção de água dos agregados empregados e fração granulométrica (maior teor de finos reduz a retração de secagem).

Assim é possível completar que, os fatores que influenciam na retração de secagem são a porosidade das argamassas (quanto maior a quantidade de poros, maior o encolhimento) e 0 módulo de elasticidade (quanto menor a rigidez do material, maior a contração gerada na mesma tensão). Geralmente, uma maior instabilidade dimensional é negativa para a durabilidade das argamassas de revestimento (M0BILI et al., 2016; LUCAS et al., 2016).

Argane et al. (2016) concluíram que a perda de água pelos poros capilares é o principal fator que afeta a retração de argamassas, no entanto, há importantes influências de outros parâmetros, tais como adições minerais e aditivos químicos nas propriedades de retração por secagem das argamassas.

A retração por secagem das amostras de argamassa geralmente é aumentada com 0 aumento do teor de água. De fato, um aumento na relação água/cimento não apenas fornece maior teor de água livre suscetível à evaporação, mas também faz com que sua evaporação seja mais fácil devido à estrutura mais porosa das argamassas (TAN e DU, 2013; JOSHAGHANI et al., 2018).

Correlacionando o estudo de Mobili et al. (2016) e Joshaghani et al. (2018), onde ambos observaram que o módulo de elasticidade influencia na retração por secagem das argamassas, as amostras com maior relação água/cimento obtiveram menor resistência, módulo de elasticidade e apresentaram uma maior tendência para a retração.

Ergenç et al. (2018) e Li et al. (2018) monitoraram a retração por 180 dias. Ergenç et al. (2018) analisaram que com o tempo, a porosidade da fissura gerada pela retração sofre autocura que pode ser atribuído às propriedades auto-curativas de argamassas à base de cal. As fissuras de retração podem surgir da tensão gerada durante a secagem quando a falta de água durante a mistura e/ou quando a quantidade de agregado não são suficientes. 
Ao verificar a influência da temperatura externa na retração de secagem nas argamassas de revestimento, foi possível concluir, que o aumento da temperatura causou a evaporação da água livre, o que resulta em um aumento da retração. Além disso, foi verificado que quanto maior a umidade relativa do ar, menor é a retração de secagem, sendo que as amostras submetidas à umidade relativa de 90\% apresentaram menores tendências de retração, podendo ajudar na prevenção da evaporação da água das argamassas (JOSHAGHANI et al., 2018).

De forma similar, Joshaghani et al. (2018) relatam que em temperaturas mais baixas $\left(25^{\circ} \mathrm{C}\right)$ geralmente produzem uma diminuição na retração de secagem devido à evaporação mais lenta. Em contraste, a água tem alta energia térmica disponível para conduzir a evaporação a temperaturas mais altas $\left(65^{\circ} \mathrm{C}\right)$. Os efeitos diretos do aumento temperatura são o aumento da taxa de evaporação da água e das tensões ocasionadas por retração. No entanto, o aumento na temperatura ajuda, na mudança de fase da água, acelerando o processo de hidratação. 0 desenvolvimento da hidratação não só pode impedir a evaporação de água livre, mas também pode aumentar a resistência à compressão e módulo de deformação, o que pode reduzir os valores de tensão de retração.

Beushausen et al. (2018) apresentaram um modelo analítico simplificado para prever a idade das fissuras de argamassas à base de cimento Portland, com base no módulo de elasticidade, tensão de retração e resistência à tração. Os autores concluíram que argamassas com baixa resistência mecânica à tração apresentaram melhor desempenho de fissuração. Sendo assim, deve-se evitar o uso da resistência mecânica à tração como indicador do desempenho de fissuração de materiais cimentícios.

Foi verificado, conforme a tabela 5, que os artigos analisados, obtiveram os resultados de retração através de diferentes metodologias, normas, parâmetros e procedimentos. Com isso, é possível concluir a importância de uma padronização dos ensaios de retração.

Tabela 5. Normas relacionadas nos artigos

\begin{tabular}{ccc}
\hline Artigo & Normas empregadas \\
\hline 01 & ASTM C 596/2017 & $\begin{array}{c}\text { Standard Test Method for drying shrinkage of mortar } \\
\text { containing hydraulic cement }\end{array}$ \\
\hline 02 & UNI EN 12617-4/2003 & $\begin{array}{c}\text { Prodotti e sistemi per la protezione e la riparazione delle } \\
\text { strutture di calcestruzzo - Metodi di prova - Determinazione } \\
\text { del ritiro e dell'espansione }\end{array}$ \\
\end{tabular}




\begin{tabular}{|c|c|c|}
\hline 03 & ASTM C 157/ 2017 & $\begin{array}{c}\text { Standard Test Method for Length Change of Hardened } \\
\text { Hydraulic-Cement Mortar and Concrete }\end{array}$ \\
\hline 04 & ASTM C 1148/ 2014 & $\begin{array}{l}\text { Standard Test Method for Measuring the Drying Shrinkage } \\
\text { of Masonry Mortar }\end{array}$ \\
\hline 05 & ASTM C1698/ 2014 & $\begin{array}{l}\text { Standard Test Method for Autogenous Strain of Cement } \\
\text { Paste and Mortar }\end{array}$ \\
\hline 06 & ASTM C1148-92/2014 & $\begin{array}{l}\text { Standard Test Method for Measuring the Drying Shrinkage } \\
\text { of Masonry }\end{array}$ \\
\hline 07 & BS EN 1015-12/2016 & $\begin{array}{c}\text { Methods of test for mortar for masonry. Determination of } \\
\text { adhesive strength of hardened rendering and plastering } \\
\text { mortars on substrates }\end{array}$ \\
\hline 08 & ASTM C 596/ 2017 & $\begin{array}{c}\text { Standard Test Method for drying shrinkage of mortar } \\
\text { containing hydraulic cement }\end{array}$ \\
\hline 09 & EN 13454-2/2003 & $\begin{array}{l}\text { Binders, composite binders and factory made mixtures or } \\
\text { floor screeds based on calcium sulfate. Test methods }\end{array}$ \\
\hline 10 & IS: 4031 (Parte 10)/1988 & $\begin{array}{l}\text { Indian Standard Methods of physical tests for hydraulic } \\
\text { cement: Determination of drying shrinkage }\end{array}$ \\
\hline 11 & ASTM C 1579/2006 & $\begin{array}{c}\text { Standard Test Method for Evaluating Plastic Shrinkage } \\
\text { Cracking of Restrained Fiber Reinforced Concrete (using a } \\
\text { steel form insert) }\end{array}$ \\
\hline 12 & EN $13454-2 / 2003$ & $\begin{array}{l}\text { Binders, composite binders and factory made mixtures or } \\
\text { floor screeds based on calcium sulfate. Test methods }\end{array}$ \\
\hline \multirow[b]{2}{*}{13} & ASTM C 157/ 2017 & $\begin{array}{c}\text { Standard Test Method for Length Change of Hardened } \\
\text { Hydraulic-Cement Mortar and Concrete }\end{array}$ \\
\hline & ASTM C 1609/C 1609M-05 & $\begin{array}{c}\text { Standard test method for flexural performance of } \\
\text { fiberreinforced concrete (using beam with third-point } \\
\text { loading) }\end{array}$ \\
\hline 14 & CAHIER 2669-4/1993 & $\begin{array}{l}\text { CSTB certification monolayer coatings for waterproofing. } \\
\text { Test procedures (in French). Scientific and Technical Centre } \\
\text { of Building }\end{array}$ \\
\hline
\end{tabular}




\begin{tabular}{|c|c|c|}
\hline 15 & ASTM C596/2017 & $\begin{array}{c}\text { Standard Test Method for drying shrinkage of mortar } \\
\text { containing hydraulic cement }\end{array}$ \\
\hline 16 & ASTM C596/2017 & $\begin{array}{c}\text { Standard Test Method for drying shrinkage of mortar } \\
\text { containing hydraulic cement }\end{array}$ \\
\hline 17 & RILEM, 1997 & $\begin{array}{l}\text { Recommendations: Part 6-thermal strain. Effect of fire } \\
\text { exposure on cracking, spalling and residual strength of fly } \\
\text { ash geopolymer concrete }\end{array}$ \\
\hline 18 & BS ISS0 1920-8 & $\begin{array}{l}\text { Determination of drying shrinkage of concrete for samples } \\
\text { prepared in the field or in the laboratory }\end{array}$ \\
\hline 19 & ASTM C596/2017 & $\begin{array}{c}\text { Standard Test Method for drying shrinkage of mortar } \\
\text { containing hydraulic cement }\end{array}$ \\
\hline 20 & CAHIER 2669-4/1993 & $\begin{array}{l}\text { CSTB certification monolayer coatings for waterproofing. } \\
\text { Test procedures (in French). Scientific and Technical Centre } \\
\text { of Building }\end{array}$ \\
\hline 21 & ASTM C596/2017 & $\begin{array}{c}\text { Standard Test Method for drying shrinkage of mortar } \\
\text { containing hydraulic cement }\end{array}$ \\
\hline 22 & ASTM C 157/ 2017 & $\begin{array}{c}\text { Standard Test Method for Length Change of Hardened } \\
\text { Hydraulic-Cement Mortar and Concrete }\end{array}$ \\
\hline 23 & & Ensaio não normatizado \\
\hline
\end{tabular}

CSTB certification monolayer coatings for waterproofing.

24 CAHIER 2669-4/1993 Test procedures (in French). Scientific and Technical Centre of Building.

25

Ensaio não normatizado

26 ASTM C531/2005

Standard Test Method for Linear Shrinkage and Coefficient of Thermal Expansion of Chemical-Resistant Mortars, Grouts, Monolithic Surfacings, and Polymer Concretes

Fonte: Autores (2020).

Verificando a tensão de retração das argamassas através de sensores de fibra óptica, Bao et al. (2015) concluíram que a tensão de retração aumentou rapidamente no início da cura 
e tornou-se estável após 7 dias. Da mesma maneira, Tan e Du (2013) analisam que a estabilidade da retração ocorre aos 21 dias. Com isso, podemos definir a importância das medições da retração nas primeiras horas da argamassa de revestimento.

\section{Análise da retração plástica em argamassas}

Foi observado que a retração plástica é pouco estudada em argamassas de revestimento, tendo poucos estudos que realizem ensaios desse tipo de retração. Os primeiros artigos do ranking não fazem referência ao estudo de retração plástica, apenas de retração de secagem, evidenciando que há poucos trabalhos relevantes em relação à retração plástica em argamassas de revestimento. Com isso, foram selecionados os artigos que abordam o estudo de retração plástica, conforme demonstrado na Tabela 6.

Tabela 6. Artigos que relatam o estudo de retração plástica

\begin{tabular}{cccc}
\hline \multirow{2}{*}{ Ranking } & Autores e títulos dos artigos & No de & Ano \\
& & & \\
\hline $12^{\mathbf{0}}$ & Fuller, A., Stegmães \\
& H., Knödler, F., Maier, J. and Scheffknecht, G. & & 2018 \\
\hline $23^{\mathbf{0}}$ & Duran, A., Navarro-Blasco, I., Fernández, J. and Alvarez, J. & 20 & 2014 \\
\hline $25^{\circ}$ & Anna, A. and Giuseppe, C. & 10 & 2014
\end{tabular}

Fonte: Autores (2020).

Conforme o estudo realizado por Fuller et al. (2018), onde a retração foi avaliada segundo as recomendações das normas EN 13454-2 e DIN 52450-1985-08, foi possível verificar que as adições empregadas nas argamassas têm influência na retração plástica. Isso ocorre devido à reatividade das adições, sendo que a retração plástica de uma argamassa aumenta com 0 aumento do grau de hidratação.

Em estudo realizado por Duran et al. (2014), os autores avaliaram tanto a retração plástica como a de secagem, sendo as medidas de retração obtidas através da variação de comprimento de amostras prismáticas de cada argamassa, em diferentes dias de cura: 1, 3, 7, 14 e 28 dias. As argamassas estudadas apresentaram maiores valores médios de retração nas primeiras horas e menores acréscimos nas medições de retração por secagem. Isso reforça que grande parcela da retração total ocorre já no estado plástico, não podendo ser negligenciado. 
Além disso, a utilização nanosílica contribuiu na redução de retração de secagem, visto que a água livre passa a ser quimicamente combinada devido às reações que ocorre.

Anna e Giuseppe (2014) analisaram a retração plástica através de um dispositivo que mede as variações dimensionais horizontais argamassa fresca, por meio de dois sensores conectados a um computador, e concluiu que embora algumas argamassas estudadas tenham originado um aumento na retração plástica, na retração de secagem foi reduzida, reforçando a importância do estudo da retração plástica das argamassas.

Efeito da incorporação de materiais alternativos e adições minerais na retração das argamassas

Li et al., (2018), Le et al., (2018), Chen e Poon (2017) e Aydin (2013) avaliaram o efeito de adições minerais na retração das argamassas, em substituição parcial do teor de cimento. Através dos resultados, os autores puderam concluir que as adições aumentaram notavelmente a retração, devido ao aumento de mesoporos. Também avaliaram que a retração por secagem reduz o grau de hidratação, consequentemente aumenta a porosidade capilar.

Kim et al. (2016) avaliaram a retração autógena nas argamassas com de agregados em pó e leves artificiais e concluíram que o pó, mesmo em pequena quantidade, foram suficientes para eliminar a retração autógena das argamassas de alta resistência.

Abraham et al. (2018) analisaram a influência da adição de fração fina do Pavimento Asfáltico Reciclado (RAP) na retração de secagem e concluíram que as misturas de argamassa com pavimento asfáltico reciclado sofreram maior retração e foi maior nos primeiros dias de secagem ao ar. Ao passo que Ralegaonkar et al. (2018) verificaram que as fibras de basalto reduzem a retração ainda no estágio plástico e Li et al. (2018) concluíram que adição de pó de granito diminui a retração de secagem, que justifica pelo fato da redução do teor de pasta de cimento e teor de água, ambos responsáveis a retração.

Silva et al. (2014) verificaram a adição de resíduos plásticos e estudo dos efeitos na incorporação em argamassas e concluíram que para o ensaio de retração por secagem houve uma redução à medida que a proporção de resíduo aumentava.

Penacho et al. (2014), Ling e Poon (2017), Pan et al. (2017) e Oliveira et al. (2015) verificaram a influência da incorporação de resíduos de vidro na retração nas argamassas. Para Penacho et al. (2014) as taxas de substituição abaixo de 50\%, a retração é semelhante à argamassa de referência, porém, foi observado altos níveis de retração para altas taxas de incorporação de resíduos de vidro (maiores que 50\%), atingindo um aumento de 19\%. Pan et al. (2017) e Oliveira 
et al. (2015) também observaram que a adição de pó de vidro resultou em maior retração, em comparação com amostras de referência.

Diferentemente de Penacho et al. (2014), Pan et al. (2017) e Oliveira et al. (2015), o estudo de Ling e Poon (2017) indicaram que o aumento do teor de vidro de lâmpadas fluorescentes resultou em uma redução na retração de secagem da argamassa, aumentando a trabalhabilidade, que pode ser atribuído à absorção de água próxima de zero.

Bayat et al. (2018) incorporaram lama vermelha nas argamassas e avaliaram a retração por secagem. As argamassas com lama vermelha apresentaram um volume cumulativo de poros e assim, concluíram que retração por secagem foi maior nas amostras com teor de lama vermelha.

Para Muhammad e Ismail (2013) a redução de volume observada em misturas de cimento é provavelmente uma das ameaças para a durabilidade das argamassas, pois geralmente é acompanhado por fissuras e, assim, concluiu que a incorporação de materiais alternativos à base de poli (1,4-isopreno) poderia inibir a formação e a propagação de fissuras ativas.

\section{CONCLUSÃO}

Diante dos fatos apresentados, apesar dos esforços empregados, não há qualquer padronização nos procedimentos e parâmetros de ensaio relacionados à retração por secagem ou retração plástica de argamassas.

Entre os fatores que influenciam a retração, alguns já são bem compreendidos (forma das amostras, umidade relativa, temperatura), outros são intrínsecos ao material que está em estudo (relação água/aglomerado, tipo de cimento, adições, agregados).

Ainda que a retração plástica represente uma parcela significativa na retração total do material, a inexistência de normas propicia o descuido com a avaliação desse comportamento. As normas, por sua vez, precisam ser criadas com embasamentos científicos, sendo resultado de diversos avanços na pesquisa. Uma vez que a retração plástica se apresentou uma frente de pesquisa bastante escassa, o que dificulta a elaboração de uma norma, tornando-se, dessa forma, um círculo vicioso.

Para a elaboração de uma norma, todos os fatores mencionados devem ser considerados, assim como a facilidade ou dificuldade de execução dos procedimentos e a disponibilidade e custo dos equipamentos. Porém, é muito importante que essas escolhas resultem em dados que remetam à realidade. 
Diante do exposto, para o entendimento do fenômeno de retração, ainda existem muitas lacunas de conhecimento que devem ser preenchidas. Vê-se na união de esforços da comunidade acadêmica um elevado potencial para o esclarecimento do fenômeno.

\section{REFERÊNCIAS BIBLIOGRÁFICAS}

ABRAHAM, S.M.; RANSINCHUNG, G. Influence of RAP aggregates on strength, durability and porosity of cement mortar. Construction and Building Materials, vol. 189, pp. 1105-1112, 2018.

ANNA, A.; GIUSEPPE, C. The water transfer properties and drying shrinkage of aerial lime-based mortars: an assessment of their quality as repair rendering materials. Environmental Earth Sciences, Vol. 71, pp. 1699-1710, 2014.

ARGANE, R., BENZAAZOUA, M., HAKKOU, R., BOUAMRANE, A. A comparative study on the practical use of low sulfide base-metal tailings as aggregates for rendering and masonry mortars. Journal of Cleaner Production, vol. 112, pp. 914-925, 2016.

ASSOCIAÇÃO BRASILEIRA DE NORMAS TÉCNICAS. NBR 15261 - Argamassa para assentamento e revestimento de paredes e tetos - Determinação da variação dimensional (retração ou expansão linear). Rio de Janeiro, 2005.

AYDIN, S. A ternary optimisation of mineral additives of alkali activated cement mortars. Construction and Building Materials, vol. 43, pp. 131-138, 2013.

BAO, Y.; MENG, W.; CHEN, Y.; CHEN, G.; KHAYAT, K.H. Measuring mortar shrinkage and cracking by pulse pre-pump Brillouin optical time domain analysis with a single optical fiber. Materials Letters, vol. 145, pp. 344-346, 2015.

BASTOS, P. K. X.; NAKAKURA, E. H. H.; CINCOTTO, M. A. Comparação da retração de argamassas industrializadas e mistas de revestimento nos estados frescos e endurecidos. Santa Catarina: Simpósio Brasileiro de Tecnologia de Argamassas, VI, 2005.

BAYAT, A., HASSANI, A.; YOUSEFI, A. Effects of red mud on the properties of fresh and hardened alkali-activated slag paste and mortar. Construction and Building Materials, vol. 167, pp. 775-790, 2018.

BEUSHAUSEN, H., ARITO, P. The influence of mix composition, w/b ratio and curing on restrained shrinkage cracking of cementitious mortars. Construction and Building Materials, vol. 174, pp. 38-46, 2018.

CHEN, Z., PO0N, C.S. Comparative studies on the effects of sewage sludge ash and fly ash on cement hydration and properties of cement mortars. Construction and Building Materials, vol. 154, pp. 791-803, 2017.

DURAN, A., NAVARR0-BLASCO, I., FERNÁNDEZ, J., ALVAREZ, J. Long-term mechanical resistance and durability of air lime mortars with large additions of nanosilica. Construction and Building Materials, vol. 58, pp. 147-158, 2014.

ERGENC, D., GÓMEZ, L.S.; FORT, R. Crystal development during carbonation of lime-based mortars in different environmental conditions. Materials Characterization, vol. 142, pp. 276-288, 2018.

FULLER, A., STEGMAIER, M., SCHULZ, N., MENKE, M., SCHELHORN, H., KNODLER, F., MAIER, J., SCHEFFKNECHT, G. Use of wood dust fly ash from an industrial pulverized fuel facility for rendering. Construction and Building Materials, vol. 189, pp. 825-842, 2018.

JOSHAGHANI, A.; BALAPOUR, M.; RAMEZANIANPOUR, A. A. Effect of controlled environmental conditions on mechanical, microstructural and durability properties of cement mortar. Construction and Building Materials, vol. 164, pp. 134-149, 2018.

KABEER, K.S.A.; VYAS, A.K. Utilization of marble powder as fine aggregate in mortar mixes.Construction and Building Materials, vol. 165, pp. 321-332, 2018. 
KIM, H., HA, K., LEE, H. Internal-curing efficiency of cold-bonded coal bottom ash aggregate for high-strength mortar. Construction and Building Materials, vol. 126, pp. 1-8, 2016.

KONSTANTIN, K.; ZHUTOVSKY, S. Overview and Future Trends of Shrinkage Research. Materials and Structures, vol. 39, 2006.

LE, H.V., M00N, D., KIM, D.J. Effects of ageing and storage conditions on the interfacial bond strength of steel fibers in mortars. Construction and Building Materials, vol. 170, pp. 129-141, 2018.

LI, L., WANG, R., LU, Q. Influence of polymer latex on the setting time, mechanical properties and durability of calcium sulfoaluminate cement mortar. Construction and Building Materials, vol. 169, pp. 911-922, 2018.

LING, T.C., P00N, C.S. Spent fluorescent lamp glass as a substitute for fine aggregate in cement mortar. Journal of Cleaner Production, vol. 161, pp. 646-654, 2017.

LU, T. Autogenous shrinkage of early age cement paste and mortar. Delft University of Technology (Tese), 2019.

LUCAS, J., DE BRITO, J., VEIGA, R., FARINHA, C. The effect of using sanitary ware as aggregates on rendering mortars' performance. Materials \& Design, vol. 91, pp. 155-164, 2016.

MEHTA, P.K.; MONTEIRO ,P.J. Concreto Microestrutura, Propriedade e Materiais, $3^{\text {a }}$ edição. Ed. IBRACON.

MELO NET0, A. A. Estudo da retração de argamassas com cimento de escória ativada - Dissertação - Escola Politécnica da Universidade de São Paulo, São Paulo, 2002.

MOBILI, A.; BELLI, A.; GIOSUĖ, C.; BELLEZZE, T.; TITTARELLI, F. Metakaolin and fly ash alkali-activated mortars compared with cementitious mortars at the same strength class. Cement and Concrete Research, vol. 88, pp. 198$210,2016$.

MUHAMMAD, B., ISMAIL, M. Performance of hydrocarbon particles on the drying shrinkage of cement mortar. Construction and Building Materials, vol. 48, pp. 868-873, 2013.

OLIVEIRA, R., BRIT0, J., VEIGA, R. Reduction of the cement content in rendering mortars with fine glass aggregates. Journal of Cleaner Production, vol. 95, pp. 75-88, 2015.

PAGANI, R., KOVALESKI, J., E RESENDE, L. Methodi Ordinatio: a proposed methodology to select and rank relevant scientific papers encompassing the impact factor, number of citation, and year of publication. Scientometrics, pp. $1-27,2015$.

PAN, Z., TA0, Z., MURPHY, T., WUHRER, R. High temperature performance of mortars containing fine glass powders. Journal of Cleaner Production, vol. 162, pp. 16-26, 2017.

PENACHO, P., BRITO, J., VEIGA, R. M. Physico-mechanical and performance characterization of mortars incorporating fine glass waste aggregate. Cement and Concrete Composites, vol. 50, p. 47-59, 2014.

RALEGAONKAR, R., GAVALI, H., ASWATH, P.; ABOLMAALI, S. Application of chopped basalt fibers in reinforced mortar: A review. Renewable and Sustainable Energy Reviews, vol. 82, pp. 3077-3090, 2018.

SEGAT, G. T. Manifestações patológicas observadas em revestimentos de argamassa: estudo de caso em conjunto habitacional popular na cidade de Caixas do Sul (RS). Dissertação (Mestrado). UFRGS, 2005.

SILVA, A.M., BRIT0, J., VEIGA, R. Incorporation of fine plastic aggregates in rendering mortars, Construction and Building Materials, Vol. 7l, pp. 226-236, 2014.

SILVA, N. G. Avaliação da retração e da fissuração em revestimentos de argamassa na fase plástica - Tese Universidade Federal de Santa Catarina, Florianópolis, 2011.

TAN, K. H.; DU, H. Use of waste glass as sand in mortar: Part I - Fresh, mechanical and durability properties. Cement and Concrete Composites,pp. 1-27, 2013. 